\title{
Characterization of the Opp Peptide Transporter of Corynebacterium pseudotuberculosis and Its Role in Virulence and Pathogenicity
}

\author{
Pablo M. R. O. Moraes, ${ }^{1}$ Nubia Seyffert, ${ }^{1}$ Wanderson M. Silva, ${ }^{1}$ Thiago L. P. Castro, ${ }^{1}$ \\ Renata F. Silva, ${ }^{1}$ Danielle D. Lima, ${ }^{2}$ Raphael Hirata Jr., ${ }^{3}$ Artur Silva, ${ }^{4}$ \\ Anderson Miyoshi, ${ }^{1}$ and Vasco Azevedo ${ }^{1}$ \\ ${ }^{1}$ Instituto de Ciências Biológicas, Universidade Federal de Minas Gerais, Belo Horizonte 31270-901, MG, Brazil \\ ${ }^{2}$ Instituto de Ciências da Saúde, Universidade Federal da Bahia, Salvador 40210-340, BA, Brazil \\ ${ }^{3}$ Faculdade de Ciências Médicas, Universidade Estadual do Rio de Janeiro, Rio de Janeiro 21941-901, RJ, Brazil \\ ${ }^{4}$ Instituto de Ciências Biológicas, Universidade Federal do Pará, Belém 66075-110, PA, Brazil \\ Correspondence should be addressed to Vasco Azevedo; vasco@icb.ufmg.br
}

Received 7 March 2014; Accepted 16 April 2014; Published 8 May 2014

Academic Editor: Ana Lucia Nascimento

Copyright (c) 2014 Pablo M. R. O. Moraes et al. This is an open access article distributed under the Creative Commons Attribution License, which permits unrestricted use, distribution, and reproduction in any medium, provided the original work is properly cited.

\begin{abstract}
Despite the economic importance of caseous lymphadenitis (CLA), a chronic disease caused by Corynebacterium pseudotuberculosis, few genes related to the virulence of its etiologic agent have been characterized. The oligopeptide permease (Opp) transporters are located in the plasma membrane and have functions generally related to the uptake of peptides from the extracellular environment. These peptide transporters, in addition to having an important role in cell nutrition, also participate in the regulation of various processes involving intercellular signaling, including the control of the expression of virulence genes in pathogenic bacteria. To study the role of Opp in C. pseudotuberculosis, an OppD deficient strain was constructed via simple crossover with a nonreplicative plasmid carrying part of the $\alpha p p D$ gene sequence. As occurred to the wild-type, the $\triangle o p p D$ strain showed impaired growth when exposed to the toxic glutathione peptide (GSH), indicating two possible scenarios: (i) that this component can be internalized by the bacterium through an Opp-independent pathway or (ii) that there is toxicity while the peptide is extracellular. Additionally, the $\triangle o p p D$ mutant presented a reduced ability to adhere to and infect macrophages compared to the wild-type, although both strains exhibit the same potential to colonize spleens and cause injury and death to infected mice.
\end{abstract}

\section{Introduction}

Corynebacterium pseudotuberculosis is a Gram-positive bacterium and a facultative intracellular parasite that causes caseous lymphadenitis (CLA), a chronic infectious disease affecting small ruminants. The widespread occurrence and economic importance of the disease have stimulated studies on the molecular basis of virulence of this pathogen, on which information is still limited [1]. The genes of $C$. pseudotuberculosis already characterized that are involved in virulence include the pld gene, which encodes a powerful exotoxin associated with pathogen dissemination in the host $[2,3]$, and the operon fag ABCD, which is associated with iron absorption [4].
Among the molecular factors associated with the virulence of pathogenic bacteria, the peptide transporters (Opp) have been highlighted, which are multisubunit protein complexes that belong to the family of $\mathrm{ABC}$ transporters. This family uses energy generated by the hydrolysis of adenosine triphosphate (ATP) to drive the transport of lipids, peptides, and saccharides across the plasma membrane $[5,6]$. These transporters are located in the plasma membrane, and their main function is to capture peptides from the extracellular environment to serve as sources of plasma carbon and nitrogen $[5,6]$. In addition to their nutritional role, the peptides can be used by Gram-positive bacteria as signaling molecules in intercellular communication, which allows the 
bacteria to coordinate the expression of specific genes at a population level. The control of various cellular processes, including sporulation, conjugation, and virulence, has been linked to communication via signal peptides [7-9]. Several studies performed on pathogenic bacteria of the genera Staphylococcus sp., Streptococcus sp., and Mycobacterium sp. have shown that Opp mutant strains show reduced virulence $[7,10,11]$.

The Opp transporters were already characterized in some Gram-positive and Gram-negative bacteria as being composed of five protein subunits generally arranged in operons: OppA, OppB, OppC, OppD, and OppF. Modifications in the gene arrangement, the presence of more than one copy, and the fusion of these subunits have already been observed in the genomes of some bacteria [12]. Generally, the OppA subunit is responsible for the capture of peptides from the extracytoplasmic environment and the transfer of these molecules to transmembrane channel-forming proteins (OppB and OppC). The OppB and OppC subunits are responsible for the formation of the transmembrane channel through which the oligonucleotides are transported to the intracellular environment. The OppD and OppF subunits are located in the cytoplasmic portion of the bacterial membrane and are responsible for ATP hydrolysis, thus generating energy for the peptide internalization process $[6,13]$.

We studied the role of the Opp transporters in Corynebacterium pseudotuberculosis through the generation of a mutant strain with a disruption in the gene $o p p D$ caused by simple homologous recombination. The knockout of $o p p D$ causes the inactivation of the entire transport system because this gene encodes the protein responsible for providing energy to the peptide internalization process through the hydrolysis of ATP [14]. After obtaining and confirming the C. pseudotuberculosis $\triangle o p p D$ genotype, tests were performed to confirm the loss of function of the transporter. This mutant strain was also assessed for adhesion and viability in macrophages and for virulence in mice to analyze the persistence of infection and the mortality rates.

\section{Materials and Methods}

2.1. Bacterial Strains, Plasmids, and Culture Conditions. The bacterial strains used were Escherichia coli DH5 $\alpha$ (Invitrogen) and Corynebacterium pseudotuberculosis wild-type strain 1002 [15], and the plasmid used was pCR2.1-TOPO (Invitrogen). The culture media used were Luria-Bertani (LB) (HIMEDIA) for E. coli and Brain Heart Infusion (BHI) (HIMEDIA) for C. pseudotuberculosis, which were supplemented with $1.5 \%$ bacteriological agar for culture on solid medium. Both cultures were grown at $37^{\circ} \mathrm{C}$ under agitation. E. coli was grown for $18 \mathrm{~h}$, and C. pseudotuberculosis was grown for $72 \mathrm{~h}$. When necessary, the culture media were supplemented with ampicillin $(100 \mu \mathrm{g} / \mathrm{mL})$ or kanamycin $(50 \mu \mathrm{g} / \mathrm{mL})$.

2.2. Construction of the C. pseudotuberculosis $\triangle o p p D$ Strain. For the construction of $C$. pseudotuberculosis $\triangle o p p D$ by simple homologous recombination [16], a clone of a $C$. pseudotuberculosis genome library generated by D'Afonseca et al. [17] was used, which contained a fragment of the open reading frame (ORF) of the cloned $o p p D$ gene. Confirmation of the portion of the $o p p D$ gene cloned into the plasmid was performed by sequencing the fragment and subsequent sequence alignment with the C. pseudotuberculosis genome. This search was performed using the Artemis software. After the identification of the E. coli clone in the genomic library that contained the $o p p D$ fragment cloned into pCR2.1TOPO (Invitrogen), plasmid DNA extraction was performed using the Wizard Plus Maxipreps DNA Purification System (Promega). The extracted plasmid was directly transformed into C. pseudotuberculosis strain 1002 according to Dorella et al. [18]. The selection of the $o p p D$ mutant clones was performed in BHI medium supplemented with $50 \mu \mathrm{g} / \mathrm{mL}$ kanamycin. To confirm $o p p D$ inactivation by the insertion of the suicide vector, polymerase chain reaction (PCR) was performed using primers aligning with $o p p D$ as well as m13 and $\mathrm{km}$. All other molecular biology techniques were performed according to Sambrook et al. [19].

2.3. In Silico Analysis of C. pseudotuberculosis Opp. Parallel to the construction of C. pseudotuberculosis $\triangle o p p D$, an analysis of the gene content of Opp, its organization, and its similarity to other bacteria was performed using the Artemis software. The operon analyses were performed using SoftBerry (http://linuxl.softberry.com/berry. (phtml?topic=fgenesb\&group=help\&subgroup $=$ gfindb).

A similarity analysis of the genes contained in the operon was performed using basic local alignment and search tool (BLASTp) (http://blast.ncbi.nlm.nih.gov/Blast.cgi), and only the best hit was used for comparison. An analysis of the protein domains present in the subunits of the transporter was performed through the conserved domain (http://www.ncbi.nlm.nih.gov/Structure/cdd/cdd.shtml).

2.4. Growth Curves of the C. pseudotuberculosis 1002 WildType and $\triangle o p p D$ Strains in the Presence of GSH. Preinocula of C. pseudotuberculosis 1002 and $\triangle o p p D$ were prepared in $20 \mathrm{~mL}$ of BHI with $0.05 \%$ Tween 80 (Sigma). After 24 hours of growth at $37^{\circ} \mathrm{C}$, aliquots of the cultures were reinoculated $(1: 100)$ in $120 \mathrm{~mL}$ BHI supplemented with Tween 80 and incubated at $37^{\circ} \mathrm{C}$ until the initial exponential growth phase was reached $\left(\mathrm{OD}_{600 \mathrm{~nm}}=\sim 0.2\right)$. At this time, GSH (Sigma) was added at concentrations of $5 \mathrm{mM}$ and $10 \mathrm{mM}$ to two aliquots of culture, according to Green et al. [20]. The controls received no treatment with GSH. The monitoring of the growth curves was performed by $\mathrm{OD}_{600 \mathrm{~nm}}$ readings at $0,15,60,120,180,240,300$, and $360 \mathrm{~min}$ after the addition of the substrate, which is toxic to cultures. From the $\mathrm{OD}_{600 \mathrm{~nm}}$ data, growth curves were plotted comparing the wild-type and mutant strains in the presence and absence of the toxic substrate GSH. The $\mathrm{OD}_{600 \mathrm{~nm}}$ data were analyzed with GraphPad Prism v.5.0 (http://www.graphpad.com/scientific-software/prism/).

2.5. Viability of C. pseudotuberculosis 1002 and $\triangle o p p D$ in the Murine Macrophages J774. The J774 cell line was 
grown in Dulbecco's modified eagle's medium (DMEM) (Life Technologies) supplemented with $50 \mu \mathrm{g} / \mathrm{mL}$ gentamicin, $2.5 \mu \mathrm{g} / \mathrm{mL}$ fungizone, and $5 \%$ fetal bovine serum (Life Technologies) at $37^{\circ} \mathrm{C}$ in a $\mathrm{CO}_{2}$ incubator. Confluent monolayers were trypsinized at two-day intervals with a saline buffer containing $0.2 \%(\mathrm{w} / \mathrm{v})$ trypsin and $0.02 \%(\mathrm{w} / \mathrm{v})$ ethylenediaminetetraacetic acid (EDTA) (Sigma). For the adhesion assays, $500 \mu \mathrm{L}$ of cell suspension $\left(5 \times 10^{5}\right.$ cells $\left./ \mathrm{mL}\right)$ was distributed into 24-well microplates and incubated in a $\mathrm{CO}_{2}$ incubator for 48 hours until the monolayer was $95 \%$ confluent. In parallel, the C. pseudotuberculosis strains were grown in $\mathrm{BHI}$ broth and incubated for 24 hours at $37^{\circ} \mathrm{C}$. After three washes of the samples with Dulbecco's phosphate-buffered saline (Life Technologies), the strains were resuspended in DMEM medium to obtain $5 \times 10^{6} \mathrm{CFU} / \mathrm{mL}$ (multiplicity of infection $(\mathrm{MOI})=10$ bacteria per macrophage). After the interaction of the bacteria and macrophages for 1,3 , and 6 hours, aliquots of the supernatants were removed, diluted, and plated on BHI agar to count the colony-forming units per milliliter (CFU/mL). The percentage of adherent cells was determined from the sum of the CFUs in the supernatant plus the CFUs associated with monolayers (CFUs associated with monolayers/CFUs in supernatant + CFUs associated with monolayers $\times 100$ ). The intracellular viability assays were performed with the addition of gentamycin after incubation times of 1,3 , and 6 hours. The percentage of viable intracellular bacteria was determined from the control of associated bacteria (without exposure to gentamicin). The significant difference among the groups was determined using the ANOVA and Tukey's tests of the GraphPad Prism 5.0 (http://www.graphpad.com/scientific-software/prism/). The value of $P<0.05$ was used as the threshold for significance.

2.6. Infection and Persistence Assays of C. pseudotuberculosis 1002 and $\triangle o p p D$. The standardization of parameters such as the lethal dose (LD50), volume of the cultures to be inoculated, most appropriate inoculation location, and intervals between immunizations were performed according to Ribeiro et al. [15]. All procedures with animals were carried out according to the regulations of the Ethics Committee for Animal Experimentation of the Federal University of Minas Gerais, Brazil. For experiments evaluating virulence through experimental infection, 6- to 8-week-old BALB/c mice were divided into three groups of 15 animals each. One group was used as a negative control and inoculated with $0.9 \%$ saline solution. The second group was infected with the $C$. pseudotuberculosis 1002 strain, and the third was infected with C. pseudotuberculosis $\triangle o p p D$. All animals were infected intraperitoneally with a final volume of $100 \mathrm{~mL}$ for each dose. The inoculations with both strains contained $10^{6} \mathrm{CFU} / \mathrm{mL}$. The animals were evaluated daily for the formation of abscesses at the site of injection, the degree of prostration, and the number of deaths. The survival rates of the animals were calculated and represented in GraphPad Prism v.5.0 (http://www.graphpad.com/scientific-software/prism/) using the Kaplan-Meier survival function. For infection persistence assays, we proceeded in the same manner as described above, except that the inoculation dose was slightly lower than the previous one $\left(5 \times 10^{5} \mathrm{CFU} / \mathrm{mL}\right)$, to prevent the animals from dying before the completion of the experiment. During the period of 1-5 days after infection, three animals from each group were sacrificed, and their spleens were removed and macerated in $2 \mathrm{~mL}$ of $0.9 \%$ saline solution. From this macerate, $10^{-1}$ and $10^{-2}$ dilutions were plated on $\mathrm{BHI}$ plates for the mice infected with the wild-type strain and on BHI supplemented with $50 \mathrm{mg} / \mathrm{mL}$ of kanamycin (Sigma) for the mice infected with the mutant strain. The results of the C. pseudotuberculosis CFU count in the spleen were calculated and represented in GraphPad Prism v.5.0 (http://www.graphpad.com/scientific-software/prism/) using the two-way ANOVA test.

\section{Results and Discussion}

3.1. Generation and Confirmation of C. pseudotuberculosis $\triangle o p p D$. With the mapping of a genomic library constructed by D'Afonseca et al. [17], it was possible to identify and sequence in one of the clones a 1,200 bp fragment from the ORF of the $O P p D$ gene ligated into the plasmid pCR2.1TOPO. The nucleotide sequence of the chromosomal DNA fragment of C. pseudotuberculosis ligated into the plasmid pCR2.1-TOPO in one of the clones of the genomic library of C. pseudotuberculosis had $100 \%$ similarity to $1,000 \mathrm{bp}$ of the $\operatorname{spp} D$ (see Figure S1 in Supplementary Material available online at http://dx.doi.org/10.1155/2014/489782). After transformation into C. pseudotuberculosis, the mutant strain showed resistance to kanamycin, and their DNA was subjected to PCR with various primer combinations (Table S1).

3.2. In Silico Characterization of the opp Operon of C. pseudotuberculosis. Through in silico analysis, we found that the Opp transporter is part of the core of C. pseudotuberculosis, and it likely has an essential role in the biology of the organism. A sequence analysis of the genes encoding the protein subunits of the Opp also showed that they are part of an operon and are transcribed in a single transcriptional unit (Figure 1). In an in silico protein analysis, a protein domain of OppA was found to be similar to an extracellular peptidebinding domain, which may be related to the capture of peptides from the extracytoplasmic environment and the transfer of these peptides to the transmembrane channel-forming proteins. Moreover, the amino acid sequences of OppB and OppC showed conserved domains related to the formation of transport channels across the plasma membrane. With respect to OppD, two protein domains related to ATP hydrolysis (Figure S2) were found, which may indicate a fusion between the $o p p D$ and $o p p F$ genes and explain the absence of the oppF gene in the operon in C. pseudotuberculosis. This same configuration was found in Mycobacterium tuberculosis, demonstrating that this fusion may be a characteristic of actinobacteria [6].

3.3. Phenotypic Test to Evaluate the Peptide Internalization of $C$. pseudotuberculosis $\triangle o p p D$. One method by which to phenotypically characterize mutant strains defective in the transport of molecules across the plasma membrane 


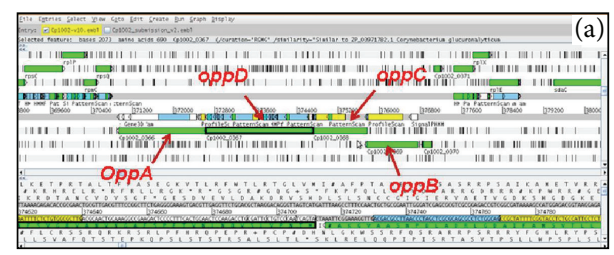

(a)

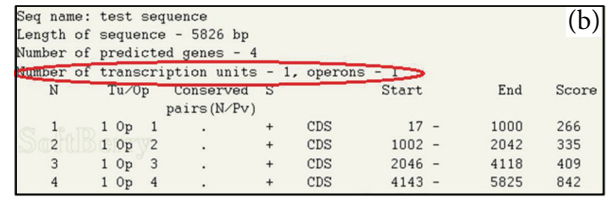

(b)

FIGURE 1: In silico analysis of the operon $o p p B C D A$ in the genome of C. pseudotuberculosis. (a) Organization of operon (visualized by software Artemis). Arrows indicate the arrangement of genes in the genome. (b) Prediction of $o p p B C D A$ operon by software FGENESB. The red circle indicates that the genes are transcribed into a single transcriptional unit.

is to use substrate analogs that are toxic to the bacterium when internalized by these transporters. This methodology is effective because it is expected that the absence of the transporter in the mutant strain makes this strain resistant to the effects of the toxic substrate given that the substrate will not be internalized by the bacterium. Conversely, in the wild-type strain, the presence of the functional transporter will enable the toxic substrate to be transported into the cell, thus causing damage to the bacterium. The use of GSH as a toxic substrate proved to be an effective tool in the characterization of mutant strains for the Opp peptide transporter in Mycobacterium bovis (phylogenetically close to C. pseudotuberculosis) [20]. Thus, we used the same molecule for the phenotypic characterization of $C$. pseudotuberculosis $\triangle o p p D$, and the results are shown in Figure 2. Unlike the study by Green et al. [20] on Mycobacterium bovis $\triangle o p p D$, in which the wild-type strain was sensitive to concentrations of $5 \mathrm{mM}$ of GSH, C. pseudotuberculosis was resistant to the toxic effects of GSH at this concentration because the wildtype and mutant strains showed the same growth profile in the presence and absence of the toxic substrate (Figure 2(a)). With the aim of finding a GSH concentration in which $C$. pseudotuberculosis was sensitive to the toxic effects of this substrate, various concentrations of GSH were tested (data not shown), and the concentration chosen was $10 \mathrm{mM}$ given that concentrations above this value inhibited the growth of both strains. Figure 2(b) shows that although GSH is toxic to C. pseudotuberculosis at the concentration of $10 \mathrm{mM}$, the use of this substrate did not allow us to phenotypically distinguish the wild-type and mutant strains because the growth of both was affected in the same way by the presence of GSH at that concentration. Two hypotheses were raised from these results: (i) GSH does not need to be transported to the cytoplasm of $C$. pseudotuberculosis to be toxic to the bacterium or (ii) GSH is being internalized by the bacterium through a pathway different from Opp.

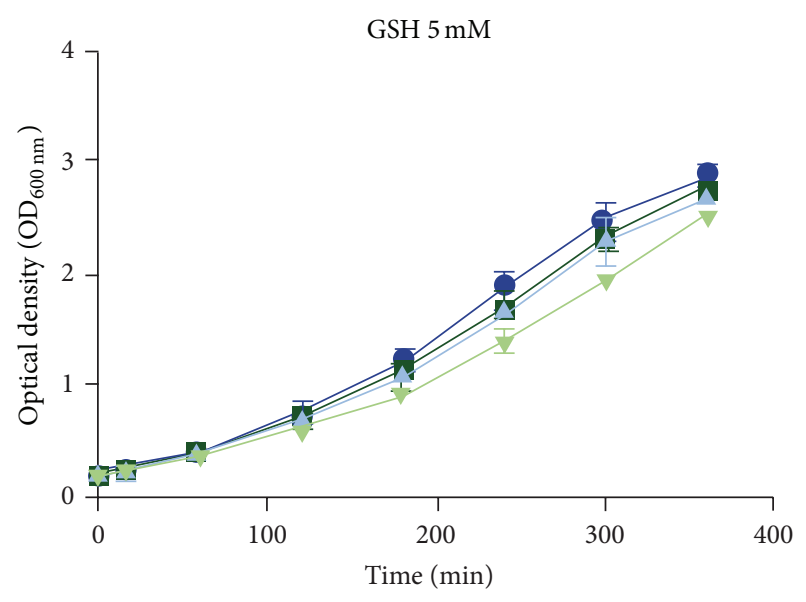

(a)

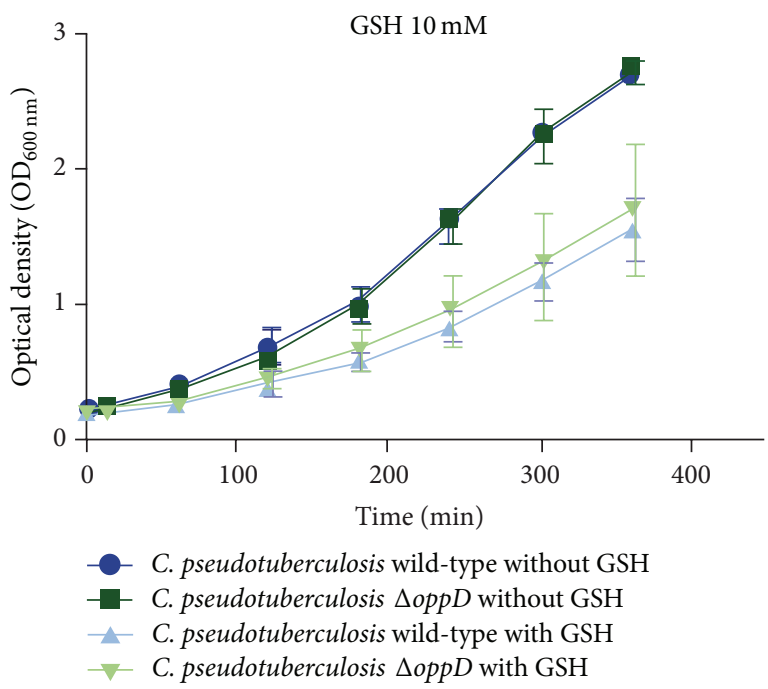

(b)

FIGURE 2: Growth of wild-type and $\triangle o p p D$ C. pseudotuberculosis in the presence or absence of GSH. (a) Phenotypic test with $5 \mathrm{mM} \mathrm{GSH}$ and (b) phenotypic test with $10 \mathrm{mM} \mathrm{GSH}$.

3.4. Infection of Macrophages by C. pseudotuberculosis $\triangle o p p D$. In the course of the infection by $C$. pseudotuberculosis, the bacterium needs to be internalized to survive in this environment as an intracellular parasite. The tests performed to evaluate whether these characteristics are still present in C. pseudotuberculosis $\triangle o p p D$ showed a significant reduction in adhesion only during the first hour, and at 3 and 6 hours, both strains showed the same ability to adhere to and infect macrophages (Figure 3(a)). These results suggest that C. pseudotuberculosis $\triangle o p p D$ exhibits a delay in the ability to adhere to the cell membranes of macrophages because only during the initial phases of the experiment the percentage of adherent mutant bacteria was lower than that of the wild-type bacteria. The lower adhesion potential of the mutant strain also led to a decline in internalization (Figure 3(b)) given that, to infect the phagocytic cell, the bacteria must first establish contact with the macrophage membrane. Studies conducted on different species of the genus Streptococcus demonstrated 


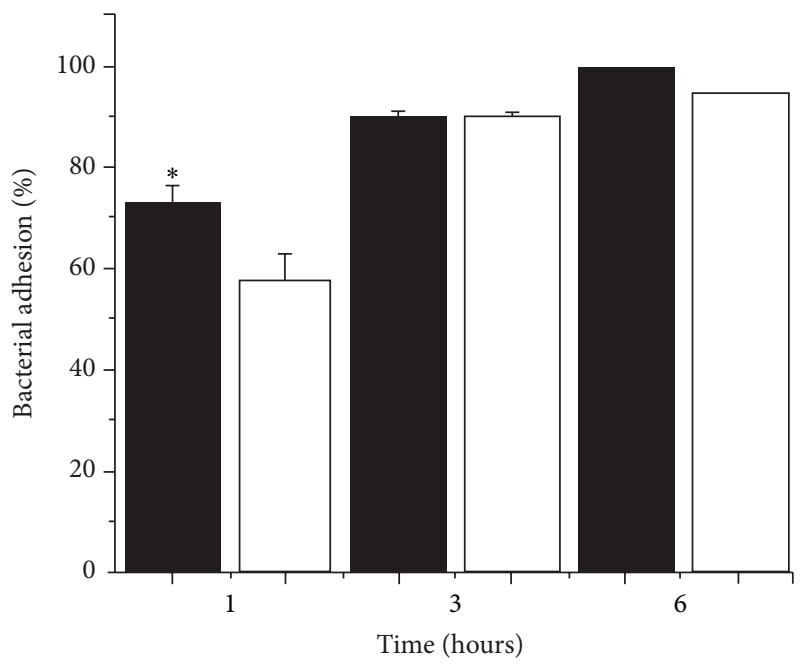

(a)

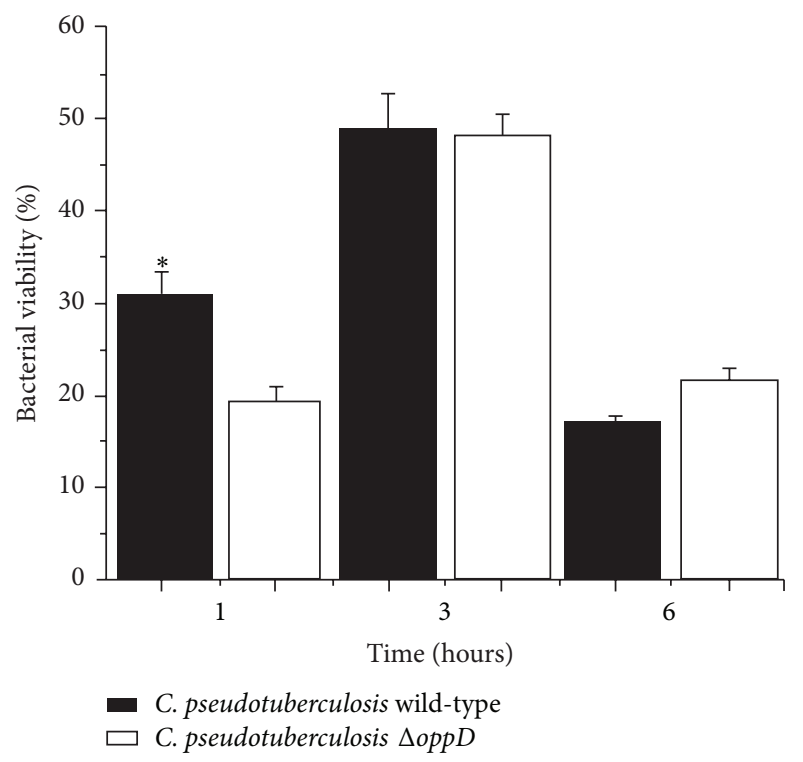

(b)

Figure 3: Adhesion and internalization of wild-type C. pseudotuberculosis and $\triangle o p p D$ mutant into J774 macrophages. (a) Bacterial adherence of wild-type and mutant strains was evaluated 1, 3, and 6 hours after infection. (b) Viability of wild-type and mutant strains inside macrophages was evaluated 3 and 6 hours after infection. Asterisks indicate significant differences between groups tested $(P<$ 0.05).

that Opp mutant strains showed a reduced ability to adhere to human cells $[21,22]$. This reduction in Streptococcus pyogenes was caused by a decrease in the expression of $f b s A$, which encodes a fibrinogen-binding adhesin [9]. Thus, Opp may be associated with the transcriptional control of genes related to bacterial adhesion.

3.5. Virulence of C. pseudotuberculosis $\triangle o p p D$ in $B A L B / c$ Mice. To assess whether the inactivation of the $o p p D$ gene in $C$. pseudotuberculosis resulted in a decrease in the virulence profile of the mutant strain, infection assays using BALB/c mice were performed. Figure 4 shows that there was no

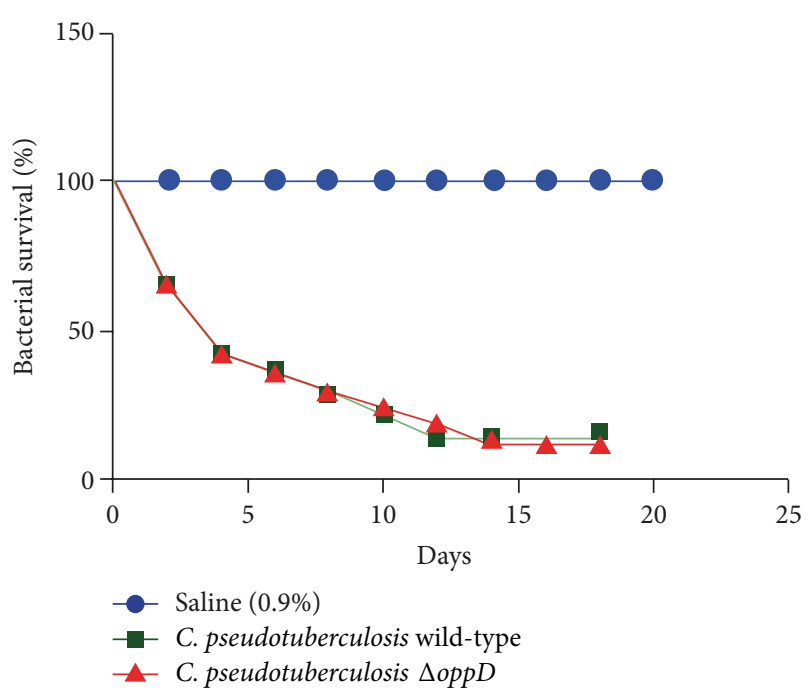

FIGURE 4: Survival curve of BALB/c mice infected with C. pseudotuberculosis $\triangle o p p D$.

significant difference between the survival curves of animals infected with C. pseudotuberculosis 1002 and those infected with $C$. pseudotuberculosis $\triangle o p p D$, corroborating the general physical examination of the mice that also showed no difference between the infected groups. This same result was observed by Flores-Valdez et al. [23] in a study performed with Mycobacterium tuberculosis in which the virulence of an Opp mutant strain was evaluated through infection of $\mathrm{BALB} / \mathrm{c}$ mice via aerosol. That study revealed that the mutant strain showed no significant difference in the ability to cause death in mice compared to the wild-type strain. However, when evaluating the colonization potential of the mutant strain in organs such as the spleen and lung during the acute, chronic, and persistent phases of infection, a lower bacterial load was observed during the chronic phase of infection in mice inoculated with this mutant strain. Unlike infections caused by $M$. tuberculosis in mice, in infections by $C$. pseudotuberculosis, it is only possible to recover live bacteria from the spleen of infected mice six days after inoculation. Thus, the assays designed to evaluate the ability to colonize the spleen of mice infected with C. pseudotuberculosis cover only the acute phase. To assess if there was a reduction in the potential for spleen colonization during the infection of mice with C. pseudotuberculosis $\triangle o p p D$, a comparison was performed between the number of CFUs of the wild-type and mutant strains recovered from the spleens of the mice during the acute phase of infection. Figure 5 demonstrates that the bacterial load found in the spleens of mice infected with the mutant strain was not significantly different from that found in animals infected with the wildtype strain. Borezee et al. [24], in a study performed with Listeria monocytogenes $\triangle o p p A$, observed a reduced growth capacity of this bacterium in murine macrophages compared to the wild-type strain. Despite this reduction, the mutant strain showed the same ability as the wild-type strain to kill the infected mice. Unlike the results of studies with other pathogenic bacteria such as $S$. aureus, M. tuberculosis, and $S$. 


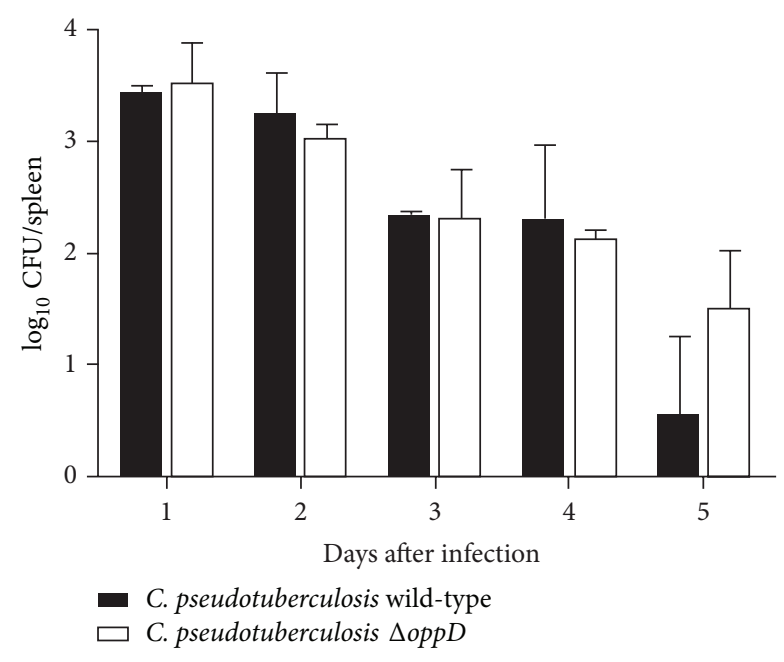

FIGURE 5: Colony-forming units in the spleen of BALB/c mice infected with wild-type C. pseudotuberculosis and $\triangle o p p D$ mutant for the first 5 days of infection.

pyogenes, in which the Opp mutant strains showed reductions in virulence and pathogenicity in assays of mice infection $[10$, $11,24]$, in C. pseudotuberculosis, the presence of a functional Opp transporter appears not to be required for the virulence of this bacterium in this infection model.

\section{Conclusions}

This study is the first report on the role of the Opp transporter in bacteria of the genus Corynebacterium spp. We characterized the Opp operon and evaluated its importance in the virulence and pathogenicity of C. pseudotuberculosis. The phenotypic tests using GSH showed that Opp is not necessary for the internalization of peptides, which can use an alternative pathway. Although the in vitro assays of bacterial adhesion and viability in murine macrophages demonstrate that C. pseudotuberculosis $\triangle o p p D$ exhibits a delay in the adhesion to the cell membrane compared to the wild-type strain, this delay did not affect the virulence of the $\triangle o p p D$ strain in mice.

\section{Conflict of Interests}

The authors declare that there is no conflict of interests regarding the publication of this paper.

\section{Authors' Contribution}

Pablo M. R. O. Moraes and Nubia Seyffert contributed equally to this work.

\section{Acknowledgment}

This project received financial support from CAPES (Coordenação de Aperfeiçoamento de Pessoal de Nível
Superior) and CNPq (Conselho Nacional de Desenvolvimento Científico e Tecnológico).

\section{References}

[1] F. A. Dorella, L. G. C. Pacheco, N. Seyffert et al., "Antigens of Corynebacterium pseudotuberculosis and prospects for vaccine development," Expert Review of Vaccines, vol. 8, no. 2, pp. 205-213, 2009.

[2] A. L. M. Hodgson, K. Carter, M. Tachedjian et al., "Efficacy of an ovine caseous lymphadenitis vaccine formulated using a genetically inactive form of the Corynebacterium pseudotuberculosis phospholipase D," Vaccine, vol. 17, no. 7-8, pp. 802-808, 1999.

[3] L. H. Williamson, "Caseous lymphadenitis in small ruminants," The Veterinary clinics of North America, vol. 17, no. 2, pp. 359371, 2001.

[4] S. J. Billington, P. A. Esmay, J. G. Songer, and B. H. Jost, "Identification and role in virulence of putative iron acquisition genes from Corynebacterium pseudotuberculosis," FEMS Microbiology Letters, vol. 208, no. 1, pp. 41-45, 2002.

[5] V. Monnet, "Bacterial oligopeptide-binding proteins," Cellular and Molecular Life Sciences, vol. 60, no. 10, pp. 2100-2114, 2003.

[6] M. Braibant, P. Gilot, and J. Content, "The ATP binding cassette (ABC) transport systems of Mycobacterium tuberculosis," FEMS Microbiology Reviews, vol. 24, no. 4, pp. 449-467, 2000.

[7] S. N. Coulter, W. R. Schwan, E. Y. W. Ng et al., "Staphylococcus aureus genetic loci impacting growth and survival in multiple infection environments," Molecular Microbiology, vol. 30, no. 2, pp. 393-404, 1998.

[8] F. J. M. Detmers, F. C. Lanfermeijer, and B. Poolman, "Peptides and ATP binding cassette peptide transporters," Research in Microbiology, vol. 152, no. 3-4, pp. 245-258, 2001.

[9] U. Samen, B. Gottschalk, B. J. Eikmanns, and D. J. Reinscheid, "Relevance of peptide uptake systems to the physiology and virulence of Streptococcus agalactiae," Journal of Bacteriology, vol. 186, no. 5, pp. 1398-1408, 2004.

[10] C.-H. Wang, C.-Y. Lin, Y.-H. Luo et al., "Effects of oligopeptide permease in group A streptococcal infection," Infection and Immunity, vol. 73, no. 5, pp. 2881-2890, 2005.

[11] C. M. Sassetti and E. J. Rubin, "Genetic requirements for mycobacterial survival during infection," Proceedings of the National Academy of Sciences of the United States of America, vol. 100, no. 22, pp. 12989-12994, 2003.

[12] A. Hiron, E. Borezée-Durant, J.-C. Piard, and V. Juillard, "Only one of four oligopeptide transport systems mediates nitrogen nutrition in Staphylococcus aureus," Journal of Bacteriology, vol. 189, no. 14, pp. 5119-5129, 2007.

[13] F. A. Quiocho and P. S. Ledvina, "Atomic structure and specificity of bacterial periplasmic receptors for active transport and chemotaxis: Variation of common themes," Molecular Microbiology, vol. 20, no. 1, pp. 17-25, 1996.

[14] C. F. Higgins, "ABC transporters: from microorganisms to man," Annual Review of Cell Biology, vol. 8, pp. 67-113, 1992.

[15] D. Ribeiro, F. Rocha, K. M. Leite et al., "An iron-acquisitiondeficient mutant of Corynebacterium pseudotuberculosis efficiently protects mice against challenge," Veterinary Research, vol. 45, article 28, 2014.

[16] V. S. Kalogeraki and S. C. Winans, "Suicide plasmids containing promoterless reporter genes can simultaneously disrupt and create fusions to target genes of diverse bacteria," Gene, vol. 188, no. 1, pp. 69-75, 1997. 
[17] V. D’Afonseca, F. Prosdocimi, F. A. Dorella et al., "Survey of genome organization and gene content of Corynebacterium pseudotuberculosis," Microbiological Research, vol. 165, no. 4, pp. 312-320, 2010.

[18] F. A. Dorella, E. M. Estevam, P. G. Cardoso et al., "An improved protocol for electrotransformation of Corynebacterium pseudotuberculosis," Veterinary Microbiology, vol. 114, no. 3-4, pp. 298-303, 2006.

[19] J. Sambrook, E. F. Fritsch, and T. Maniatis, Molecular Cloning: A Laboratory Manual, Cold Spring Harbor Laboratory, Cold Spring Harbor, NY, USA, 2nd edition, 1989.

[20] R. M. Green, A. Seth, and N. D. Connell, "A peptide permease mutant of Mycobacterium bovis BCG resistant to the toxic peptides glutathione and S-nitrosoglutathione," Infection and Immunity, vol. 68, no. 2, pp. 429-436, 2000.

[21] D. R. Cundell, B. J. Pearce, J. Sandros, A. M. Naughton, and H. R. Masure, "Peptide permeases from Streptococcus pneumoniae affect adherence to eucaryotic cells," Infection and Immunity, vol. 63, no. 7, pp. 2493-2498, 1995.

[22] G. L. Darmstadt, L. Mentele, A. Podbielski, and C. E. Rubens, "Role of group A streptococcal virulence factors in adherence to keratinocytes," Infection and Immunity, vol. 68, no. 3, pp. 12151221, 2000.

[23] M. A. Flores-Valdez, R. P. Morris, F. Laval, M. Daffé, and G. K. Schoolnik, "Mycobacterium tuberculosis modulates its cell surface via an oligopeptide permease (Opp) transport system," FASEB Journal, vol. 23, no. 12, pp. 4091-4104, 2009.

[24] E. Borezee, E. Pellegrini, and P. Berche, "OppA of listeria monocytogenes, an oligopeptide-binding protein required for bacterial growth at low temperature and involved in intracellular survival," Infection and Immunity, vol. 68, no. 12, pp. 70697077, 2000. 


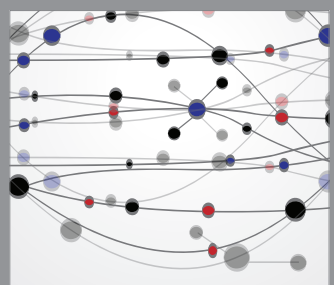

The Scientific World Journal
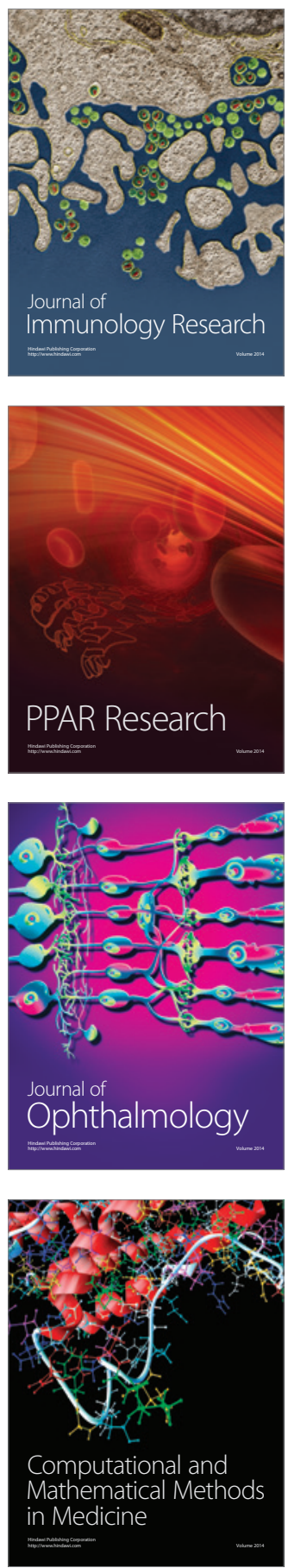

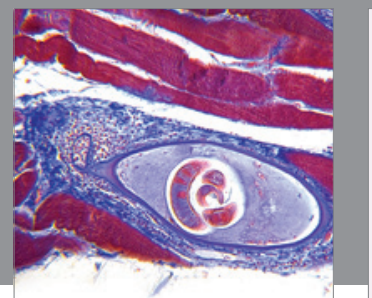

Gastroenterology

Research and Practice
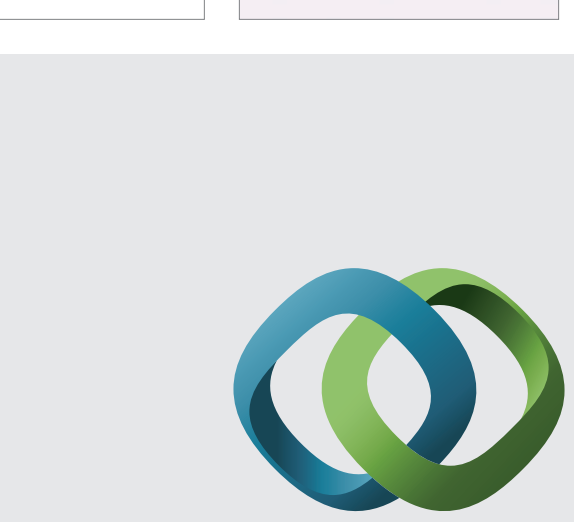

\section{Hindawi}

Submit your manuscripts at

http://www.hindawi.com
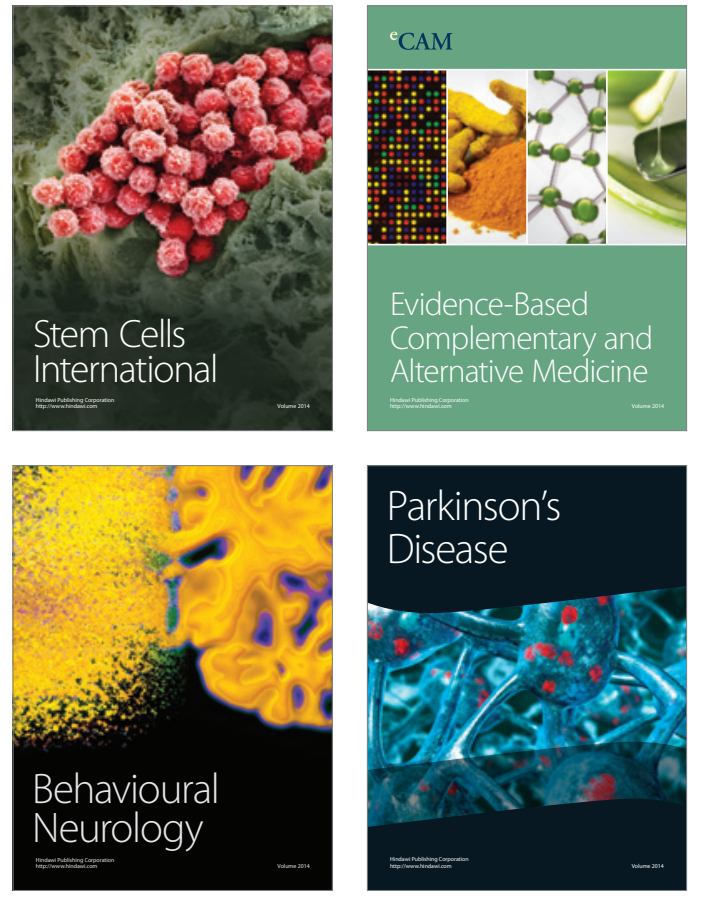
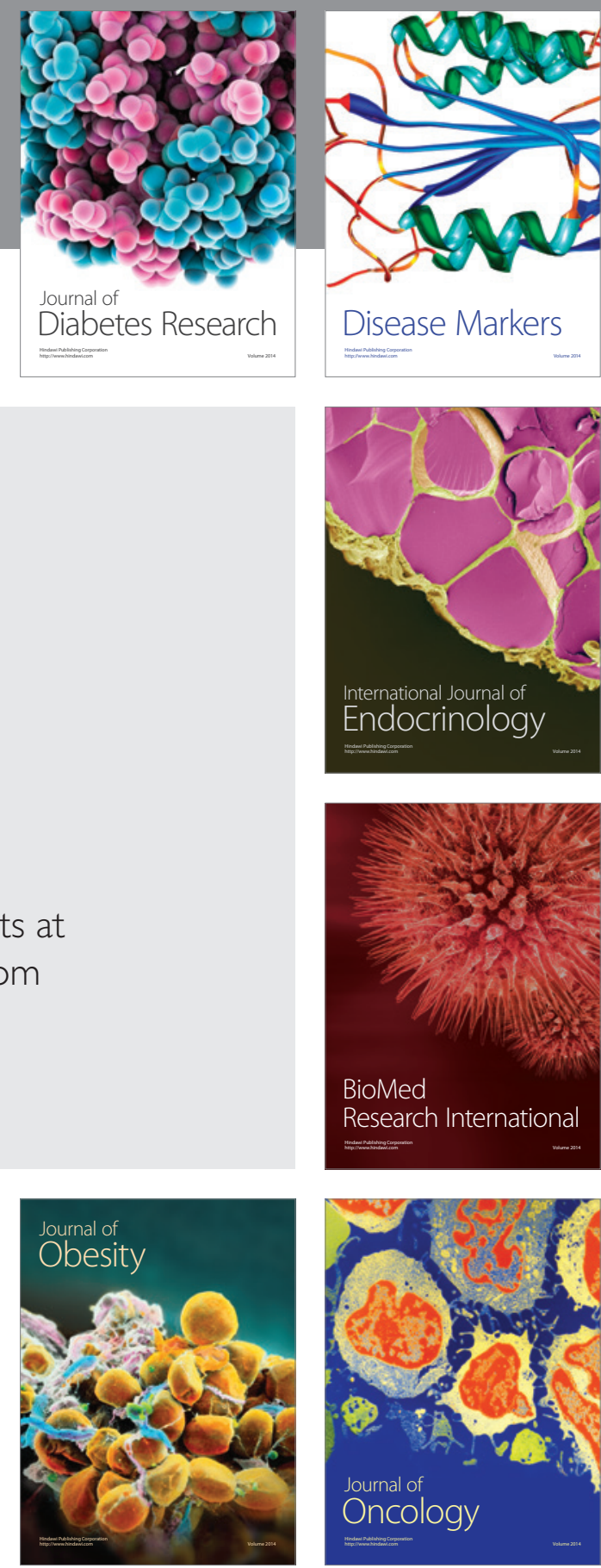

Disease Markers
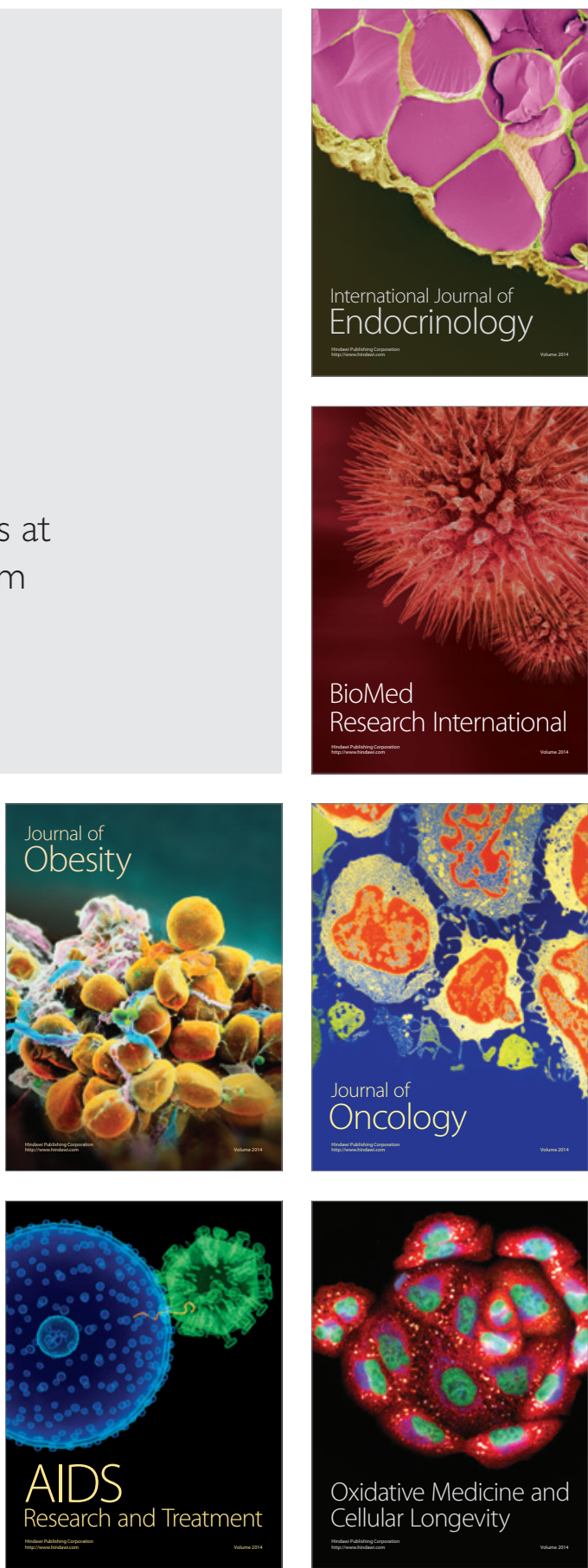